\title{
Design of One Dimensional Defective Photonic Crystal Selective Filters
}

\author{
Ouarda Barkat ${ }^{1}$, Badreddine Mamri ${ }^{1}$ \\ Electromagnetism And Telecommunications Laboratory, Department Of Electronics, \\ University Frères Mentouri Constantine, Algeria
}

\begin{abstract}
In this paper, we present a design and analysis of one dimensional defective photonic crystal (1D$D P C)$. The study of photonic crystals with defects is a topic of great interest. It is a structure built of three different dielectrics. The defect layer was situated in the middle of the dielectric layers. The proposed asymmetric and symmetric structures are simulated using the transfer matrix method. The simulated results are concerning the transmission coefficient for both TE and TM modes. The effects of angle of incidence and polarization on the transmission spectra are investigated as the function of number of defect layers. Also, simulation results obtained showing the number of defect mode within the photonic band gap (PBG) in the asymmetric (1D-DPC) structure, more than in the symmetric (1D-DPC) structure. Results are compared with previously published data and are found to be in good agreement.
\end{abstract}

Keywords: Photonic band gap, transfer matrix method, defect mode, transmission

\section{Introduction}

The one dimensional photonic crystal (1D- PC) structures have a number of useful properties, which are employed as low-loss optical waveguides, dielectric reflecting mirrors, optical switches, optical limiters, and optical filters etc [1]-[5]. The advantages of these structures are structurally simple and easy to be manufactured. The selective filters can be simply realized by introducing a defect layer into the (1D- PC) structure to break the periodicity. The defect modes will be located within the (1D- PC) structures, which are much more similar to the defect states generated in the forbidden band in a doped semiconductor. Recently, the defect modes inside the photonic band have been widely studied due to their properties [6]-[10]. The perfect layer can be realized, by changing physical parameters, such as changing the thickness of one of the layer, adding another medium to the structure, or removing a layer from (1D- PC) structures. The localized defect modes, which are also called resonant transmission peaks, can be generated within the PBG due to the change of the interference behavior of light. The defect models properties can be used to make high quality extremely narrowband frequency selective filters [7], [11]. Selective photonic crystal filters have received much research interest in the fields of demultiplexers for WDM systems [12].

Various techniques has been used to study this structure, such as with transfer matrix method, finite elements, plane wave expansion method, etc. Each method has its own limitations for finding some important properties of one dimensional defective photonic crystal (1D- DPC). The transfer matrix method is most popular because of its simplicity in algorithm and capability to model complex structures [13]-[14]. It is recently introduced by Pendry and MacKinnon, to calculate the electromagnetic transmission through the PBG materials.

In this paper, the transmission spectrum was obtained by applying the transfer matrix formalism to one dimensional defective photonic crystal (1D- DPC). The effects of the polarization and the angle of incidence on the defect modes in the transmission spectra are investigated. Several simulation cases by Matlab will be given 
to show the performance of this approach. The accuracy of the analysis is tested by comparing the computed results with measurements published data.

\section{Theory}

The (1D-PC) structure is composed of alternating multilayer of the form $(A B)^{N} D(A B)^{N}$ and $(A B)^{N} D(B A)^{N}$ shown in Fig 1, there are $2 \mathrm{~N}+1$ layer made up of dielectric materials $\mathrm{A}$ and $\mathrm{B}$, and dielectric defect layer $\mathrm{D}$. Each layer has to be $d_{1}$ thicknesses, and refractive index $n_{1}$. In order to start the computation of the (1D-PC) structure characteristics, it is necessary to define the refractive index profile of considered structure, can be given as

and

$$
\varepsilon_{l}= \begin{cases}\varepsilon_{1} & 0<z<\mathrm{d}_{1} \\ \varepsilon_{2} & \mathrm{~d}_{1}<z<\mathrm{d}_{2}\end{cases}
$$

$$
\varepsilon_{l}(\mathrm{z})=\varepsilon_{l}(\mathrm{z}+d)
$$

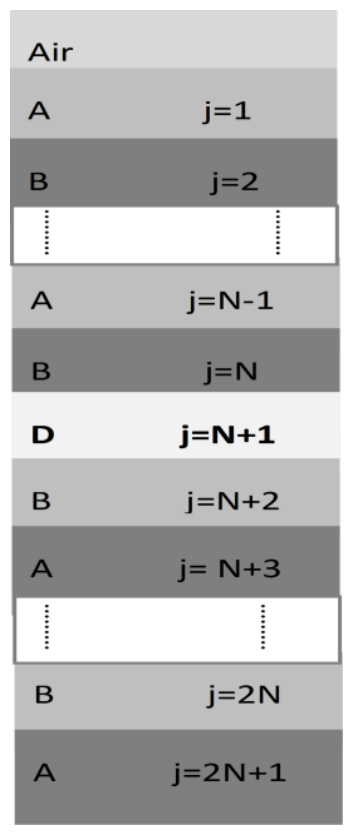

(a)

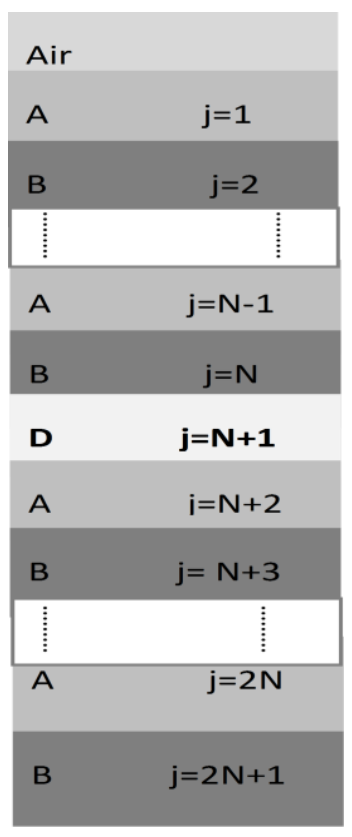

(b)

Fig. 1: Structure of one-dimensional photonic crystal containing a defect

a- Symmetric defective (1D- DPC)

b- Asymmetric defective (1D- DPC)

Where

1 : is number of layer

$\mathrm{d}=\mathrm{d}_{1}+\mathrm{d}_{2}$ : is period

Based on the plane wave propagation in different media, in terms of transfer matrices. The global transfer matrix of the stratified material is the product of all elementary transfer matrices $\left[\mathrm{M}_{\mathrm{i}}\right]$ of each layer. This matrix is given by:

$$
\left[\begin{array}{l}
E_{1} \\
H_{1}
\end{array}\right]=\mathrm{M}_{1} \mathrm{M}_{2} \ldots \ldots \mathrm{M}_{\mathrm{N}} \mathrm{M}_{\mathrm{d}} \ldots \mathrm{M}_{2 \mathrm{~N}} \mathrm{M}_{2 \mathrm{~N}+1}\left[\begin{array}{c}
E_{l} \\
H_{l}
\end{array}\right]
$$

The matrix $\mathrm{M}_{1-1}$ of the $1^{\text {th }}$ layer can be written in the form: 


$$
\mathrm{M}_{(\mathrm{l}-1)}=\left[\begin{array}{cc}
\cos \left(\delta_{(\mathrm{l}-1)}\right) & \mathrm{i} \gamma_{(\mathrm{l}-1)} \sin \left(\delta_{(l-1)}\right) \\
\mathrm{i} \gamma_{(1-1)}{ }^{-1} \sin \left(\delta_{(l-1)}\right) & \cos \left(\delta_{(l-1)}\right)
\end{array}\right]
$$

$\delta_{(l-1)}$ and $\gamma_{(l-1)}$ being the matrix parameters and depending on the incident angle of light, the optical constants and the layer thickness, are expressed as:

$$
\begin{gathered}
\delta_{(l-1)}=k_{(l-1)} \cdot d_{(l-1)} \cdot \cos \theta_{(l-1)} \\
\gamma_{(l-1)}=\left\{\begin{array}{cr}
\frac{\eta_{(l-1)}}{\cos \theta_{(l-1)}} & \text { TE mode } \\
\eta_{(l-1)} \cos \theta_{(l-1)} & \text { TM mode }
\end{array}\right.
\end{gathered}
$$

We note that $\theta_{(l-1)}$ is related to the angle of incidence $\theta_{0}$ by the Snell's Descart's low, that is

$$
n_{(l-1)} \sin \theta_{(l-1)}=n_{0} \sin \theta_{0}
$$

By considering the transmission matrix of each layer, we are able to obtain the transmission matrix of whole structure. For 1 number of multilayers; the corresponding transfer matrix can be defined as a product of matrices, is obtained for symmetric PBG structure.

$$
M=\prod_{k=1}^{(2 N+1)} M_{k}=\left(M_{A} M_{B}\right)^{N} M_{D}\left(M_{B} M_{A}\right)^{(2 N+1)}=\left[\begin{array}{ll}
\mathrm{m}_{11} & \mathrm{~m}_{12} \\
\mathrm{~m}_{21} & \mathrm{~m}_{22}
\end{array}\right]
$$

And for asymmetric PBG structure.

$$
M=\prod_{k=1}^{(2 N+1)} M_{k}=\left(M_{A} M_{B}\right)^{N} M_{D}\left(M_{A} M_{B}\right)^{(2 N+1)}=\left[\begin{array}{ll}
\mathrm{m}_{11} & \mathrm{~m}_{12} \\
\mathrm{~m}_{21} & \mathrm{~m}_{22}
\end{array}\right]
$$

Where

$\mathrm{M}_{\mathrm{A}}$ is the transfer matrix of the first dielectric layer.

$\mathrm{M}_{\mathrm{B}}$ is the transfer matrix of the second dielectric layer.

$\mathrm{M}_{\mathrm{D}}$ is the transfer matrix of the dielectric layer defect.

These formulas can be modified by using $\mathrm{N}$ number of defect, are obtained for symmetric PC.

$$
M=\left(M_{A} M_{B}\right)^{N} M_{D} \cdot M_{B} \cdot M_{D} \ldots .\left(M_{B} M_{A}\right)^{(2 N+L)}=\left[\begin{array}{ll}
\mathrm{m}_{11} & \mathrm{~m}_{12} \\
\mathrm{~m}_{21} & \mathrm{~m}_{22}
\end{array}\right]
$$

And for asymmetric PC,

$$
M=\left(M_{A} M_{B}\right)^{N} M_{D} \cdot M_{B} \cdot M_{D} \ldots .\left(M_{A} M_{B}\right)^{(2 N+L)}=\left[\begin{array}{ll}
\mathrm{m}_{11} & \mathrm{~m}_{12} \\
\mathrm{~m}_{21} & \mathrm{~m}_{22}
\end{array}\right]
$$

Where $\mathrm{m}_{11}, \mathrm{~m}_{12}, \mathrm{~m}_{21}$ and $\mathrm{m}_{22}$ are the complex numbers.

The transmittance $t$ and reflectance $r$ are defined as the ratios of the fluxes of the transmitted and reflected waves, respectively, to the flux of the incident wave. After some derivations, the total transmission and reflection coefficients are given by

$$
\begin{array}{r}
r=\frac{\left(m_{11}+p_{s}^{-1} m_{12}\right) p_{0}^{-1}-\left(m_{21}+p_{s}^{-1} m_{22}\right)}{\left(m_{11}+p_{s}^{-1} m_{12}\right) p_{0}^{-1}+\left(m_{21}+p_{s}^{-1} m_{22}\right)} \\
t=\frac{2 \cdot p_{0}^{-1}}{\left(m_{11}+p_{s}^{-1} m_{12}\right) p_{0}^{-1}+\left(m_{21}+p_{s}^{-1} m_{22}\right)}
\end{array}
$$


The reflectance $\mathrm{R}$ and transmittance $\mathrm{T}$ spectrums of can be obtained by using the expressions:

$$
\begin{aligned}
& T=|t|^{2} \\
& R=|r|^{2}
\end{aligned}
$$

\section{Numerical Calculation and Discussion}

The simulation results have been shown to validate the effectiveness of the expression proposed in this paper. We consider incidence of the electromagnetic wave on the (1D- PC) structure. The geometry of multilayer structure is shown in Figure 1. This structure is a combination of two dielectric materials $\mathrm{A}\left(\mathrm{n}_{\mathrm{GaAs}}=\right.$ $\left.3.3842, \mathrm{~d}_{\mathrm{GaAs}}=0.0332 \mu \mathrm{m}\right)$ and $\mathrm{B}\left(\mathrm{n}_{\mathrm{SiO} 2}=1.4672, \mathrm{~d}_{\mathrm{SiO} 2}=0.0732 \mu \mathrm{m}\right)$. In order to check the effects of the defect layer, we introduce a defect layer $\mathrm{TiO}_{2}\left(\mathrm{n}_{\mathrm{TiO} 2}=2.6245, \mathrm{~d}_{\mathrm{TiO} 2}=0.1433 \mu \mathrm{m}\right)$ inside of structure. Figures $2-3$ show the comparison of illustrate the transmittance spectra values of the proposed symmetric and asymmetric (1DDPC) structures. It can be seen that a very narrow pass band or single defect mode within the photonic band gap (PBG). It is noted that the bandwidth of asymmetric (1D-DPC) structure with the configuration $\left(A i r /(A B)^{4} D(A B)^{4} / A i r\right)$ is $\Delta_{\mathrm{Bwp}}=265.5 \mathrm{~nm}$, and the resonant wavelength is $\lambda=474.35 \mathrm{~nm}$. For the symmetric (1D- DPC) structure with the configuration $\left(A /(A B)^{4} D(B A)^{4} / A i r\right)$, the bandwidth was calculated as $\Delta_{\text {Bwp }}$ $=267.04 \mathrm{~nm}$, the resonant peak locates at the design wavelength of $\lambda=396.3 \mathrm{~nm}$. Also, it can clearly be seen in figures $3 \mathrm{a}-3 \mathrm{~b}$ that it has considerably reduced amplitude of the defect peak in the case of asymmetric structure as compared to the high- amplitude of the defect peak in the case of symmetric structure. These behaviors agree very well with those reported by work [13] - [15].
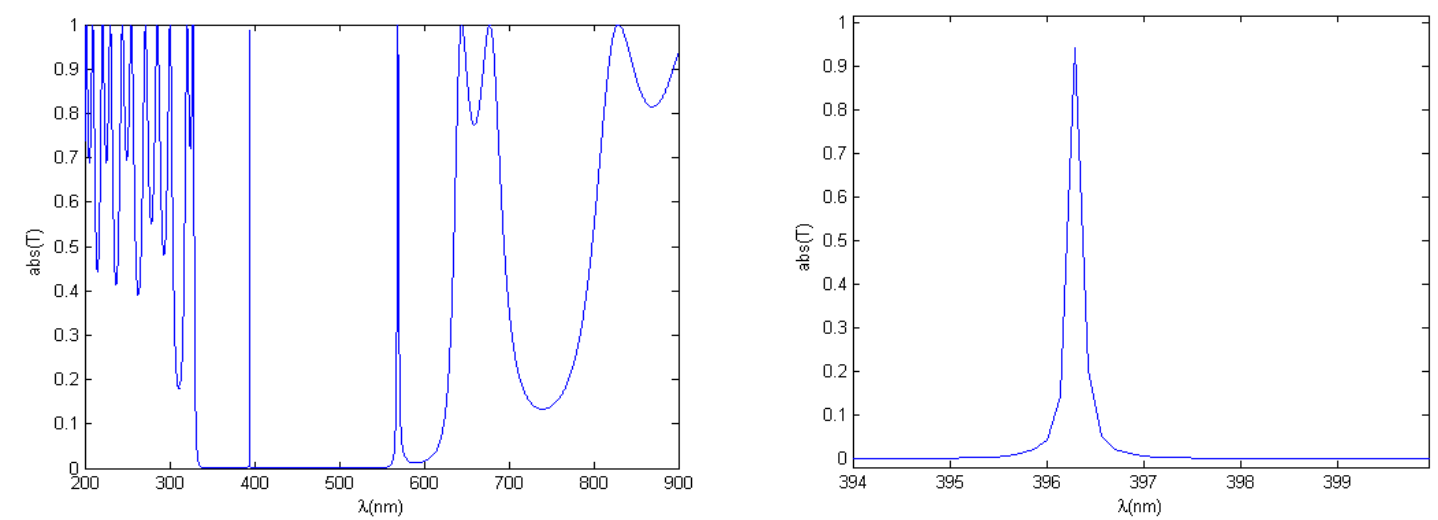

Fig. 2: Transmission spectra of symmetric (1D-DPC) structure $\left(\mathrm{n}_{\mathrm{GaAs}}=3.3842, \mathrm{n}_{\mathrm{SiO} 2}=1.4672, \mathrm{~d}_{\mathrm{GaAs}}=0.0332 \mu \mathrm{m}\right.$, $\mathrm{d}_{\mathrm{SiO} 2}=0.0732 \mu \mathrm{m}, \mathrm{n}_{\mathrm{TiO} 2}=2.6245, \mathrm{~d}_{\mathrm{TiO} 2}=0.1433 \mu \mathrm{m}, \theta=0^{\circ}$ )
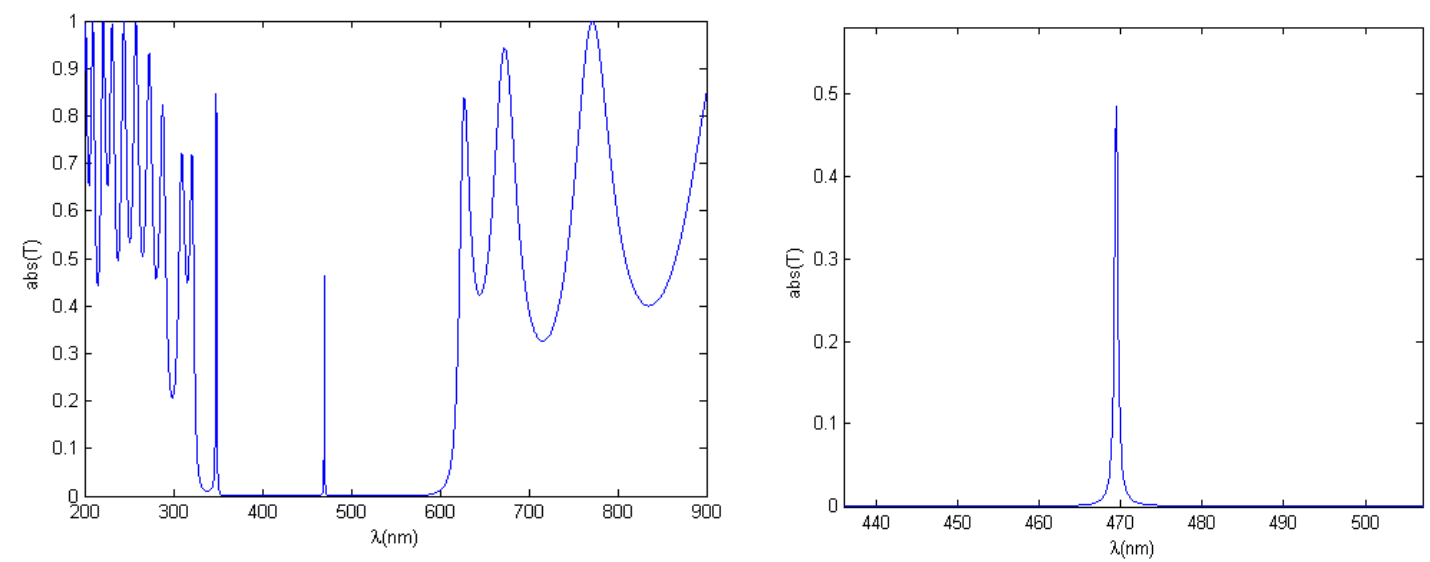

Fig. 3: Transmission spectra of asymmetric (1D-DPC) structure $\left(\mathrm{n}_{\mathrm{GaAs}}=3.3842, \mathrm{n}_{\mathrm{SiO} 2}=1.4672, \mathrm{~d}_{\mathrm{GaAs}}=0.0332 \mu \mathrm{m}\right.$, $\mathrm{d}_{\mathrm{SiO} 2}=0.0732 \mu \mathrm{m}, \mathrm{n}_{\mathrm{TiO} 2}=2.6245, \mathrm{~d}_{\mathrm{TiO} 2}=0.1433 \mu \mathrm{m}, \theta=0^{\circ}$ ) 
The effects of the polarization and the angle of incidence on the defect modes in the transmission spectra for TE or TM modes are illustrated in Table I. We have clearly observed that the transmittance spectrum of the defect mode is linked to the angle of incidence in TE and TM modes. Also, the position of narrow pass band is changed, and width of the band increases obviously for TE mode, and decrease for TM mode. On the other hand a pick of transmission change in the direction of small values when the angle incidences increase for TM mode.

TABLE I: Bandwidth and wavelength with different the angle of incidence and polarization of Transmission spectra of (1D- DPC) structure symmetric $\left(\operatorname{Air} /(A B)^{4} D(B A)^{4} / A i r\right),\left(\mathrm{n}_{\mathrm{GaAs}}=3.3842, \mathrm{n}_{\mathrm{SiO} 2}=1.4672, \mathrm{~d}_{\mathrm{GaAs}}=0.0332 \mu \mathrm{m}, \mathrm{d}_{\mathrm{SiO} 2}=0.0732\right.$ $\left.\mu \mathrm{m}, \mathrm{n}_{\mathrm{TiO} 2}=2.6245, \mathrm{~d}_{\mathrm{TiO} 2}=0.1433 \mu \mathrm{m}\right)$

\begin{tabular}{|l|c|c|c|c|}
\cline { 2 - 5 } \multicolumn{1}{c|}{} & \multicolumn{2}{c|}{ TE mode } & \multicolumn{2}{c|}{ TM mode } \\
\cline { 2 - 5 } \multicolumn{1}{c|}{} & \multicolumn{3}{c|}{} & \\
\hline$\theta$ & $\Delta_{\text {Bwp }}(\mathrm{nm})$ & $\lambda(\mathrm{nm})$ & $\Delta_{\text {Bwp }}(\mathrm{nm})$ & $\lambda(\mathrm{nm})$ \\
\hline $0^{\circ}$ & 267.04 & 396.3 & 267.04 & 396.3 \\
\hline $30^{\circ}$ & 234.7 & 384.1 & 214.2 & 386.2 \\
\hline $60^{\circ}$ & 242.36 & 359.3 & 173.5 & 364.2 \\
\hline
\end{tabular}

In Figures 4, we examine the effects of the two defect layers, by introducing two defect layer $\mathrm{TiO}_{2}$, in the (1D- DPC) structures symmetric and asymmetric. It is found that there exists a two defect mode within the photonic band gap (PBG) in the (1D- DPC) structures with the configuration $\left.\operatorname{Air} /(A B)^{4} D B D(B A)^{4} / A i r\right)$ and $\left(A \mathrm{ir} /(A B)^{4} D B D(A B)^{4} / A i r\right)$. It is noted that the bandwidth of asymmetric (1D-DPC) structure was calculated as $\Delta_{\mathrm{Bwp}}=(590.322-340,604)=249.72 \mathrm{~nm}$, as well as the resonant peak locates at the design wavelength of $\lambda_{1}$ $=390.575 \mathrm{~nm}$ and $\lambda_{2}=474.93 \mathrm{~nm}$. For the symmetric (1D- DPC) structure, we have $\Delta_{\text {bip }}=(560.871-$ $327.375)=233.49 \mathrm{~nm}$, the resonant peak locates at the design wavelength of $\lambda_{1}=369,34 \mathrm{~nm}$ and $\lambda_{2}=$ $412,76 \mathrm{~nm}$.

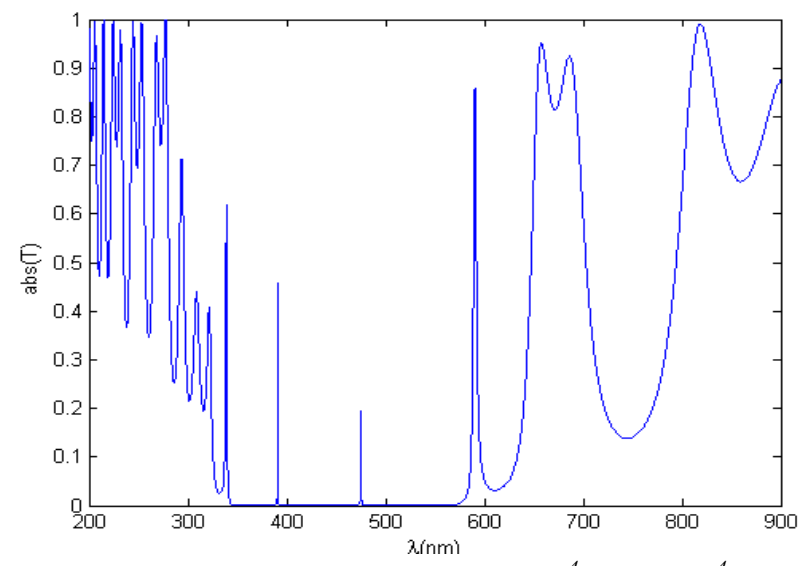

Structure asymmetric $\left(\operatorname{Air} /(A B)^{4} D B D(A B)^{4} / A i r\right)$

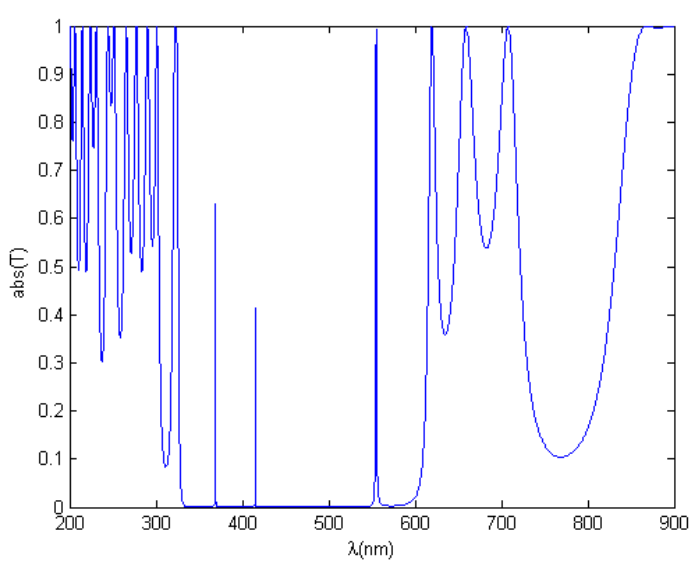

Structure symmetric $\left(\operatorname{Air} /(A B)^{4} D B D(B A)^{4} / A i r\right)$,

Fig. 4: Transmission spectra of (1D- DPC) structure symmetric $\left(A i r /(A B)^{4} D B D(B A)^{4} / A i r\right), \mathrm{n}_{\mathrm{GaAs}}=3.3842, \mathrm{n}_{\mathrm{SiO} 2}=1.4672$,

$$
\mathrm{n}_{\mathrm{TiO} 2}=2.6245, \mathrm{~N}=19 \text {. }
$$

In Figures 5, we have examined the effect the effects of the tree defect layers, by introducing tree defect layer $\mathrm{TiO}_{2}$ in the (1D- DPC) structures symmetric and asymmetric. It was found that it existed a tree defect mode within the photonic band gap (PBG) in the (1D- DPC) structure symmetric with the configuration $\left(\operatorname{Air} /(A B)^{4} D B D B D(B A)^{4} / A i r\right.$. Our results imply that the number of defect mode can be increased by adding the layer $\mathrm{TiO}_{2}$ in structure [8]. 


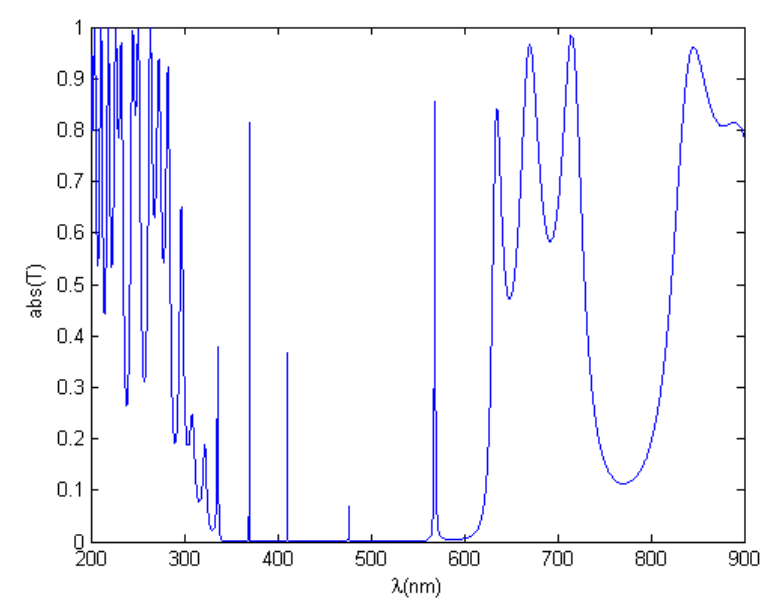

Structure asymmetric $\left(\operatorname{Air} /(A B)^{4} D B D B D(A B)^{4} / A i r\right)$

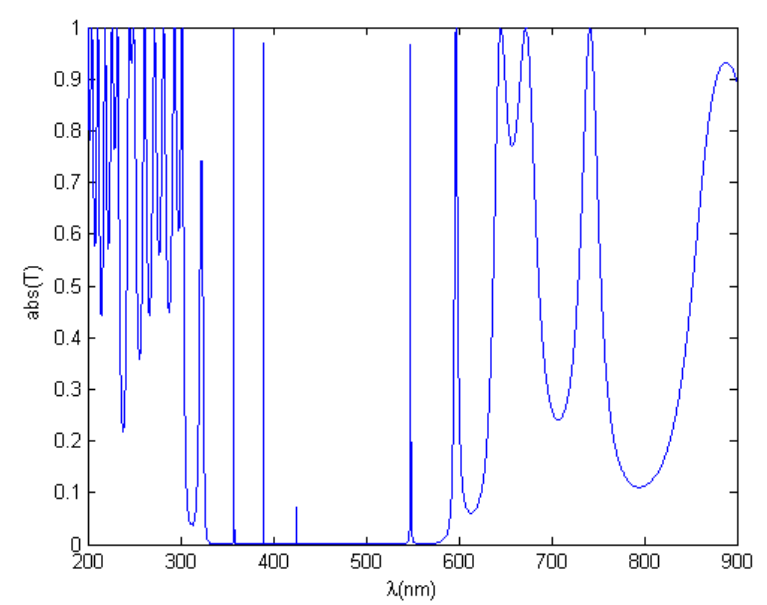

Structure symmetric $\left(\operatorname{Air} /(A B)^{4} D B D B D(B A)^{4} /\right.$ Air $)$

Fig 5 Transmission spectra of (1D- DPC) structures $\left(\mathrm{n}_{\mathrm{GaAs}}=3.3842, \mathrm{n}_{\mathrm{SiO} 2}=1.4672, \mathrm{n}_{\mathrm{TiO} 2}=2.6245, \mathrm{~N}=21\right.$.

\section{Conclusion}

In this paper, we have used the transfer matrix method to study one-dimensional photonic crystal defectives. We have discussed the properties of the defect modes for the asymmetric and symmetric structures for both TE and TM modes at different incidence angles. The results have shown the calculated the transmission with defects separated by different layers. When the order of the defect layer increased such as $m=1,2,3$ etc, additional modes occurs in photonic band gap. We can conclude that when the existence of defect layer, there is a very narrow pass band whose pick of transmission is observed. The maximum of transmission was obviously observed at these wavelengths for TE mode.

\section{References}

[1] Sakoda K, Optical properties of Photonic Crystals, New York, Springer Berlin Heidlberg, 2005.

[2] E. Gondek, P.Karasiński, "One-dimensional photonic crystals as selective back reflectors, " Optics \& Laser Technology, vol. 48, pp 438-446, June 2013.

[3] V. Kumar, B. Suthar, A. Kumar, Kh. S. Singh, A. Bhargava, S. P. Ojha, "Silicon based one-dimensional photonic crystal as a TM-mode filter," Springer science Silicon.,vol. 6, pp 73-78, January 2014.

[4] L.Young, E. G. Cristal, "Low-Pass and High-Pass Filters Consisting of Multilayer Dielectric Stacks," IEEE Transactions on Microwave Theory and Techniques., vol.14, pp 75 -80, Feb. 1966.

[5] I. V. Shadrivov, A. A. Sukhorukov, and Y. S. Kivshar, "Complete band gaps in one-dimensional left-handed periodic structures," Physical Review Letters., vol.95, pp. 1-4, 2005.

[6] S. Ya. Vetrov, A. V. Shabanov, E. V. Shustitskiı̌, "Control of the transmission spectrum of a photonic crystal with lattice defects,"Optics and Spectroscopy., Vol. 100, Issue 3, pp 409-413, March 2006.

[7] A.Hadjira. Badaoui and M. Abri , "One-dimensional photonic crystal selective filters design using simulated annealing optimization technique,"Progress in electromagnetic research B., vol.53, pp 107-125, 2013.

[8] L.Tang, L. Gao, and J. XingFang, "Characterization for defect modes of one-dimensional photonic crystals containing metamaterials,"Chinese optics letters., vol.6, N 3, March 10, 2008.

[9] E. Lotfi, K. Jamshidi-Ghaleh, F. Moslem, H. Masalehdan, "Comparison of photonic crystal narrow filters with metamaterials and dielectric defects,'The European Physical Journal D., vol.60, Issue 2, pp 369-372, November 2010.

[10] H. Němec, P. Kužel, F. Garet, and L. Duvillaret, "Time-domain terahertz study of defect formation in one-dimensional photonic crystals,"OSA Publishing, Applied Optics., vol.43, Issue 9, pp. 1965-1970, 2004. 
[11] J.A.Gaspar, "Photonic crystal to photonic crystal surface modes: Narrow-band pass filters,"Optics express., vol.12, $\mathrm{N}^{\circ} 11$, May 2004.

[12] R. Ghoch, K.K. Ghoch, R. Chakraborty, "Narrow band filter using 1D periodic structure with defects for DWDM systems,"Optics communications., pp 75-80, India, 2013.

[13] C.-J. Wu and Z.-H. Wang, "properties of defect modes in one-dimensional photonic crystals,"Progress In Electromagnetics Research, PIER 103., pp169-184, 2010.

[14] T. A. Tameh, B.I.Memarzadeh, N.Granpayeh, A. R. Maleki. Javan , "Analysis and optimization of optical bistability in one-dimensional nonlinear photonic crystal with $(\mathrm{HL})^{\mathrm{p}}(\mathrm{D})^{\mathrm{q}}(\mathrm{LH})^{\mathrm{p}}$ and $(\mathrm{LH})^{\mathrm{p}}(\mathrm{D})^{\mathrm{q}}(\mathrm{HL})^{\mathrm{p}}$ structures," Elsevier, Optik, vol. 121, pp. 1729-1734, 2010.

[15] V. Kumar, B. Suthar, A. Kumar, A. Bhargava, "Design of a wavelength division demultiplexer using Si-based onedimensional photonic crystal with a defect,"Elsevier, Optik., vol 124, pp 2527-2530, 2013. 